\title{
Rechnerunterstützte Befundung von Eiweißelektrophoresen auf Celluloseacetatfolie
}

\author{
Von W. Knüppel, D. Neumeier, A. Fateh-Moghadam und M. Knedel \\ Institut für Klinische Chemie (Direktor: Prof. Dr. med. M. Knedel) am Klinikum Großhadern \\ der Ludwig-Maximilians-Universität München
}

(Eingegangen am 30. November 1983/9. März 1984)

Zusammenfassung: In der klinisch-chemischen Routinediagnostik war eine effiziente rechnerunterstützte Befundung von Elektropherogrammen technologisch bislang nicht durchführbar. Der on-line Anschluß eines vollmechanisierten Elektrophoresegerätes an das Labordatensystem eines zentralen Institutes für Klinische Chemie ermöglicht die Durchführung und Befundung von 300 Elektrophoresen pro Tag. Dabei werden nicht nur zusätzliche Informationen zum Patienten (bestimmte klinische Diagnosen, Vorwerte) und die quantitativen Veränderungen der Elektrophoresefraktionen, sondern auch Formveränderungen der Pherogrammkurve im Albumin-, $\beta$ - und $\gamma$-Globulin-Bereich in die Auswertung miteinbezogen. Dadurch können Doppelalbuminämien differenziert sowie transitorische Albuminverbreiterungen und monoklonale Gammopathien erkannt werden. Bei Veränderungen der quantitativen Ergebnisse einzelner Fraktionen und der Gesamteiweißkonzentration werden Hinweise bei Dysproteinämien, Mangel- und Defektproteinämien ausgegeben. Die Zuordnung der Pherogrammkonstellationen zu den medizinischen Befundmustern wird über eine Nummerncodierung der graduierten quantitativen Ergebnisse gesteuert. Das gewählte Vorgehen ist effizient und auf andere Gebiete übertragbar.

\section{Computer assisted interpretative reporting for serum protein electrophoresis on cellulose acetate film}

Summary: Until a short time ago efficient computer assisted reporting of electrophoretograms was not possible owing to the lack of appropriate technology. By integrating a fully mechanized electrophoresis system into the laboratory data processing system of a centralized institute of clinical chemistry, the separation and the interpretation of results can be performed for 300 samples per day.

The interpretation is based upon available patient data (specified clinical diagnoses, previous results), calculated fractions and analysis of the electrophoretic curve in the area of albumin and the $\beta$ - and $\gamma$-globulin fraction. In this way it is possible to classify hereditary bisalbuminaemias and to detect transient bisalbuminaemias and monoclonal gammopathies. Diagnostic indications of specific dysproteinaemias and defect proteinaemias are printed on the report form if individual serum protein fractions and the total protein content are changed specifically. The results of the different electrophoretic fractions, the total protein and a set of possible alterations of the curve are numerically coded according to the scaled biochemical ranges in five definite sections or to a decision matrix (alteration 'given' or 'not given'), respectively. The composed 'result patterns' are matched with preassigned 'reference patterns' stored in the computer file. The procedure is efficient and adaptable to other areas of analysis.

\section{Einfiihrung}

Die Serumelektrophorese auf Trägermedien hat sich seit Einführung durch Grassmann und Mitarbeiter (1) zu einem Standardverfahren in der medizini- schen Diagnostik und Therapiekontrolle entwickelt. Für klinisch-chemische Routineuntersuchungen ist z.Z. keine vergleichbare analytische Methode verfügbar, die bei geringem Arbeits- und Kostenauf- 
wand mit einer Probenmenge von unter $1 \mu l$ ähnliche komplexe Information zu pathobiochemischen Veränderungen liefert. Steigende Untersuchungszahlen und der hohe manuelle Arbeitsanteil zwingen dazu, das Verfahren zu mechanisieren. Zusätzlich ist anzustreben, nicht nur die quantitativen Ergebnisse der Flächenintegrale der Serumproteinfraktionen, sondern auch wesentliche in der Pherogrammkurve enthaltene Informationen auf dem Befund auszudrukken. Algorithmen zur Ausgabe von Interpretationshilfen von Elektropherogrammen mittels Mustererkennung sind mehrfach beschrieben (2-5). Nachteile dieser Verfahren sind die häufig umständliche Codierung und der große Speicherplatzbedarf.

\section{Zur Durchführung des elektrophoretischen Verfah-} rens verwenden wir ein vollmechanisiertes Elektrophoresegerät (OLYMPUS HITE-System) ${ }^{1}$ ). Das Gerät vollzieht in der Mechanisierung das manuelle Verfahren analog nach. Über ein Rechner-Subsystem $^{2}$ ) ist es an das Labordatenverarbeitungssystem des Institutes angeschlossen. Die Verbesserungen, die mit dieser Gerätekonfiguration erzielt werden, sind eine mit manuellen Methoden nicht erreichbare analytische Präzision $(6,7)$, eine standardisierte Peakidentifikation nach der für die Serumelektrophorese gültigen Nomenklatur, eine eindeutige Probenidentifikation, eine gesicherte Zuordnung der Ergebnisse zu den demographischen Patientendaten und ein erheblicher Personalzeitgewinn (7). Daneben können die ermittelten analytischen Ergebnisse und die "on-line" erfaßten Daten zur Kurvenform weiter ausgewertet und mit gespeicherten Patientendaten verknüpft werden, um einerseits eine Plausibilitätskontrolle zu ermöglichen und andererseits dem Laborarzt und dem klinisch tätigen Kollegen eine rechnerunterstützte Interpretation der Pherogramme anzubieten.

Ziel dieser Arbeit ist es, ein Verfahren vorzustellen, das eine einfache, effiziente und speicherplatzsparende Zuordnung der quantitativen Analysenergebnisse und bestimmter Kurvenformen zu medizinischen Befundmustern erlaubt. Wir verwenden zur Festlegung von Entscheidungsgrenzen medizinische Erfahrungswerte, die z.Z. an geeigneten Patientenkollektiven statistisch überprüft werden.

\footnotetext{
1) OLYMPUS OPTICAL CO. GmbH, Hamburg.

2) Prozeßrechner SIEMENS 330, SIEMENS AG, Erlangen.
}

\section{Methodik}

Aus den Patientendaten, die im Labordatensystem gespeichert sind (demographische Daten, mitgeteilte klinische Diagnosen, Vorwerte), und den Ergebnissen der Auswertung des Elektropherogramms (Kurvenform, Relativprozent-Ergebnisse) wird ein Ergebnismuster gebildet. Dieses wird mit einer Liste korrespondierender, festgelegter Referenzmuster verglichen (Abb. 1). Die Referenzmuster entsprechen definierten, in digitale Form umgesetzten Befundkonstellationen, die vom Laborarzt mittels eines zusätzlichen Programms lediglich einmal in eine Datei eingegeben werden. Bei Úbereinstimmụng des Ergebnismusters mit einem der Referenzmuster wird die für dieses Muster zutreffende Beurteilung ausgedruckt.

Der Vorgang ist in 4 Schritte zu unterteilen:

- Auswertung des Pherogrammkurvenverlaufs

- Codierung der Ergebnisse und der Personaldaten

- Vergleich mit abgespeicherten Referenzmustern

- Ausdruck der Beurteilung

Auswertung des Pherogrammkurvenverlaufes

Die gefärbten Proteinbanden des Pherogramms werden im Densitometer optisch vermessen und die digitalisierten Absorptionswerte in das Rechnersystem "on-line" übergeben. Die Fraktionsintegrale werden errechnet. Die standardisierte Erkennung mit Zuweisung der Peaks zu den definierten Elektrophoresefraktionen erfolgt anhand der Fraktionsfenster, die durch ein Referenzserum festgelegt sind (7).

Bei der Beurteilung des Serumeiweiß-Bildes sind nicht nur die quantitativen Veränderungen der Elektrophoresefraktionen von diagnosttischer Bedeutung, sondern auch Formveränderungen der Kurve. Anhand charakteristischer Kurvenveränderungen im Albumin, $\beta$ - und $\gamma$-Globulin-Bereich können Doppelalbuminämien bzw. Paraproteinämien erkannt werden. Erst durch die von uns vorgenommene standardisierte Peakerkennung wird es möglich, den Albumin-, sowie vor allem den $\beta$ - und $\gamma$-Globulin-Bereich exakt zu definieren und in mehreren Rechnermoduln schrittweise eine weitere Auswertung.vorzunehmen.

\section{Albuminpeak}

Der Albuminpeak der Normalelektrophorese ist symmetrisch und eingipfelig. Veränderungen können sich als Doppelgipfeligkeit darstellen, wobei der zusätzliche auftretende Albuminpeak entweder eine schnelle oder langsame Wanderungsgeschwindigkeit besitzt. Diese Anomalien sind genetisch bedingt und werden als heterozygote Form einer Alloalbuminämie (Doppelalbuminämie) vom schnellen bzw. langsamen Typ̄ bezeichnet (8). Sie haben eine sehr niedrige Prävalenz von 0,0002-0,0007 und sind ohne Krankheitswert. Transitorische Formen sind dagegen durch eine anodische Verbreiterung des Albuminpeaks charakterisiert. Als mögliche Ursachen für die transitorische anodische Verbreiterung sind eine hochdosierte Antibiotikatherapie mit Penicillinen und und Cephalosporinen (9-16) sowie fistelnde, akute Pankreatitiden $(9,15-23)$ beschrieben.

Zur Erkennung und Unterscheidung der Doppelalbuminämien (Abb. 2) wird die Anzahl der vorhandenen Peaks im Albuminfen$\operatorname{ster}\left(N_{1}, N_{2}\right)$ und die Strecke vom Minimum żwischen der $\alpha_{1}$ - und $\alpha_{2}$-Globulin-Fraktion bis zum Maximum des ersten Albuminpeàks gemessen (c). Ist z. B. bei Doppelgipfeligkeit die Strecke gegenüber der Norm vergrößert, so handelt es sich um eine Doppelalbuminämie vom schnellen Typ. Zusätzlich wird die Albuminbreite am Schnittpunkt der Basislinie mit der auf halber Amplitudenhöhe angelegten Tangente der ansteigenden und abfallenden Albuminflanke errechnet (a). In 50 Seren klinisch nicht kranker Erwachsener wurde eine mittlere Albuminbreite von etwa $3 \mathrm{~mm}$ bei einer Variationsbreite $( \pm 2 \mathrm{~s})$ von \pm 9.6 Prozent gemessen. 


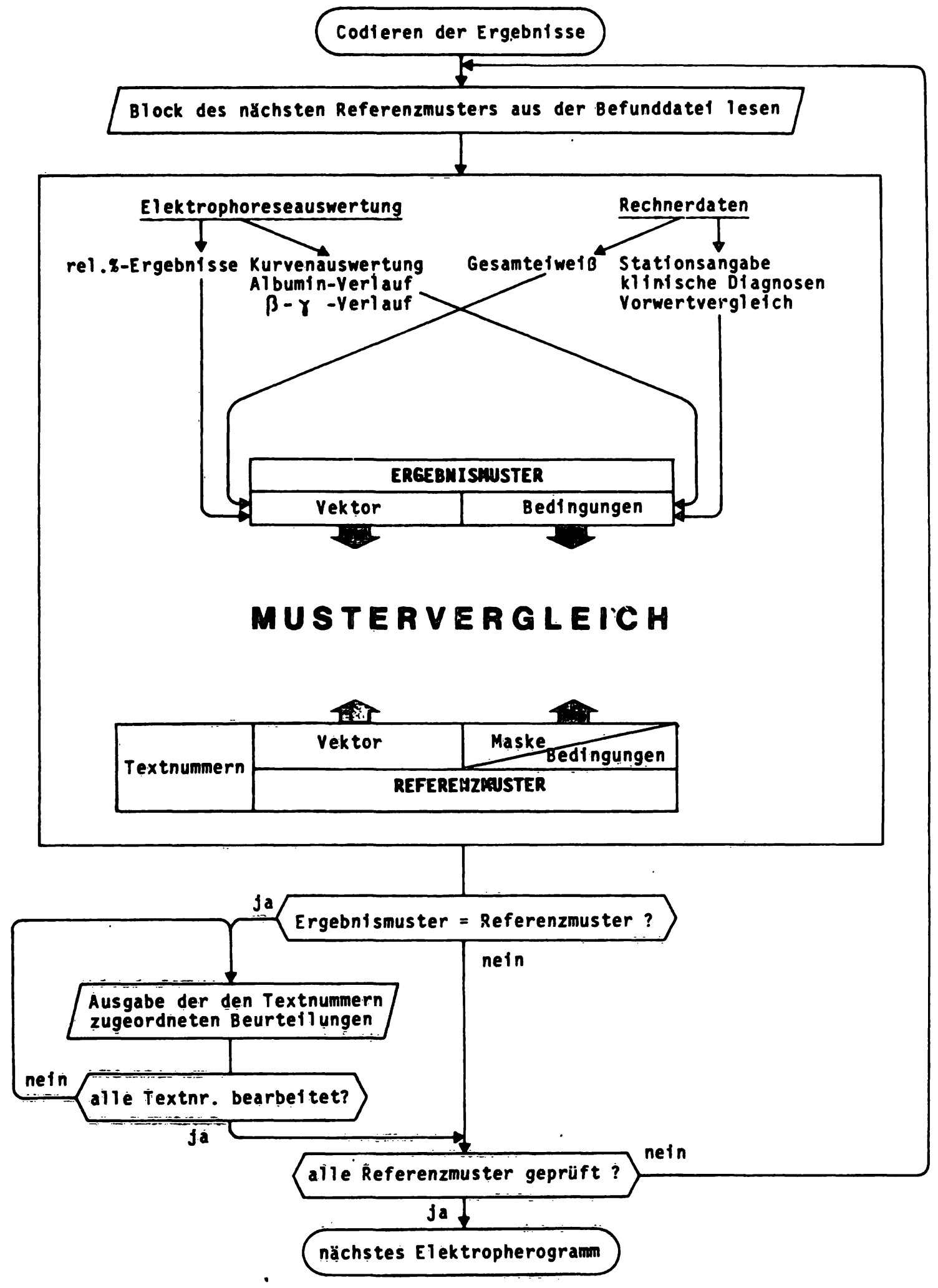

Abb. 1. Schematischer Programmablauf der Befundmustercodierung.

Kurvenverlauf im $\beta$ - und $\gamma$-Globulin-Bereich

\section{M-Peaks}

Auf die Bedeutụng der elektrophoretischen Serumtrennung zur Erkennung von monoklonalen Gammopathien bzw. Paraproteinämien wurde vielfach hingewiesen $(24,25)$. Bei einer Paraproteinämie werden von einem Zell-Klon exzessive Mengen eines Immunglobulins gebildet. In der Elektrophorese kommt es dadurch zu einem schmalbasigen Peak (M-Peak), meist im $\beta$ - und $\gamma$ -
Globulin-Bereich, selten im $\alpha_{2}$-Globulin-Bereich. Dieser monoklonale Peak wird entweder an dem Auftreten einer Sonderfraktion festgestellt, oder er wird von einer bereits vorhandenen Fraktion so überlagert, daß die schmalbasige Komponente nur an der charakteristischen Kurvenform erkannt werden kann. Ausgehend von ersten Versuchen zur Erkennung monoklonaler Gammopathien, die von uns bereits 1978 mit anderer apparativer Ausstattung durchgeführt wurden (26), wählten wir ein 2-stufiges Vorgehen. 


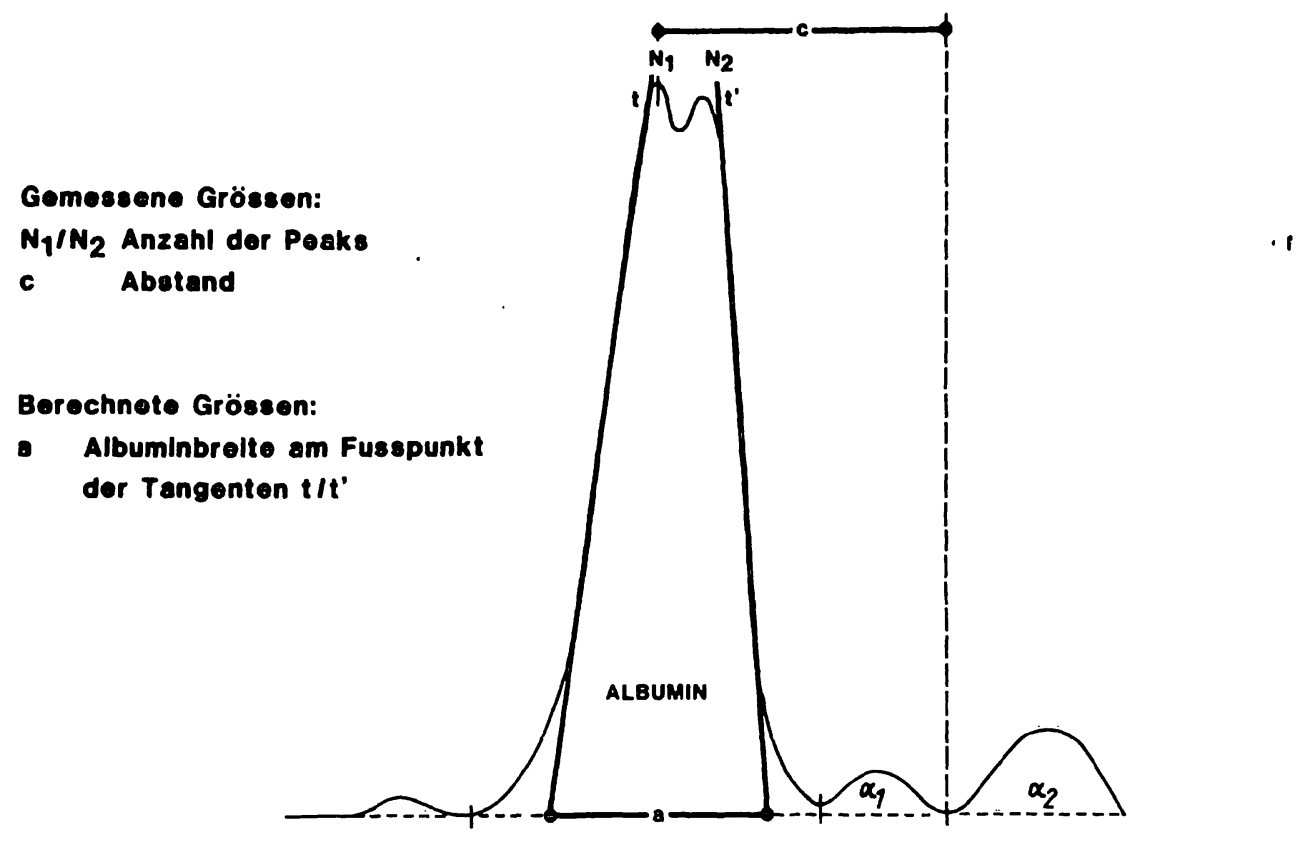

Abb. 2. Entscheidungsvariablen Doppelalbuminämien.

\section{1.a Sonderfraktionen}

In dem von uns verwendeten Elektrophoresesystem wird ein Normalserum in die Fraktionen Präalbumin, Albumin, $\alpha_{1^{-}}, \alpha_{2-}, \beta$-und $\gamma$-Globuline aufgetrennt, die von uns als Standardfraktionen bezeichnet werden. Ein zusätzlicher Peak im $\beta$-oder $\gamma$-Globulin-Bereich, eine sog. Sonderfraktion $\left(N_{1}\right)$ wird dann abgetrennt, wenn die Maxima-/Minimadifferenzen der Sonderfraktion zu beiden benachbarten Kurventälern $\left(d_{1}, d_{2}\right)$ und die Höhendifferenz des neu ermittelten Kurventales zum Peakmaximum der Standardfraktion $\left(d_{3}\right)$ jeweils einen Mindestwert überschreiten (Abb. 3a). Diese Werte wurden vorläufig auf die 0,005-fache Höhe des Albuminpeaks festgelegt. Dies entspricht nach unseren Erfahrungen einem klinisch anwendbaren Kompromiß zwischen größtmöglicher Empfindlichkeit und wünschenswerter Spezifität. Bei Erkennung von Sonderfraktionen wird in der Befundung der Verdacht auf eine monoklonale Gammopathie geäußert und die Durchführung einer Immunelektrophorese empfohlen.

\section{1.b Uberlagerte Peaks}

Weicht die Zahl der Peaks nicht von der einer Normalelektrophorese ab, so wird der $\beta$ - $\gamma$-Globulin-Bereich auf Peak-Uberlagerungen untersucht (Abb. 3b). Als geeignetes Maß kann das Höhen-/ Breitenverhältnis herangezogen werden. Als Höhe (a) ist die Läñge des Lotes vom Peakmaximum bis zum Schnittpunkt mit einer durch den Wendepunkt (W) (27) gezogenen Horizontalen festgelegt. Als Breite (b) wird die Strecke auf der Horizontalen vom Wendepunkt der Kurve bis zum Schnittpunkt mit dem Lot bezeichnet. Der ermittelte Wert wird durch die Gesamtfläche unter der Kurve (f) dividiert, da die monoklonale Gammopathie um so wahrscheinlicher ist, je kleiner - bei gleichbleibendem Höhen-/ Breitenverhältnis - die Fläche ist. Da sich die M-Peaks an jeder beliebigen Position mit einem bestehenden Peak überlagern können, werden darüberhinaus Veränderungen im Abstand des fragliçhen M-Peaks żum Albuminpeak (I) mit den Peakabständen des Referenzserums verglichen und in die Auswertung miteinbezogen.

\section{2. $\beta$ - $\gamma$-Brückenbildung (bridging)}

Weiterhin gibt es charakteristische Kurvenveränderungen in der Elektrophorese im $\beta$ - und $\gamma$-Globulin-Bereich, die nicht durch
Paraproteinämien verursacht werden. Durch eine ausgeprägte Erhöhung der Immunglobuline der Klasse A, z.B. bei toxischer Leberschädigung oder bei chronisch-entzündlichen Prozessen (28), kommt es zu einem fließenden Ubergang der $\beta$ - in die $\gamma$-GlobulinFraktion, den man als $\beta-\gamma$ Brückenbildung beżeichnet (Abb:4). Ist $b>0.8 \cdot a$, so wird dies im Ergebnismuster markiert.

\section{Codierung der Ergebnisse und der Patientendaten}

\section{Datenstruktur der Muster}

Die Beschreibung der Musterstruktur.jst zum besseren Verständnis von Mustergenerierung und -erkennung von Bedeutung.

\section{Ergebnismuster}

Das Ergebnismuster besteht aus 4 Datenwörtern (Abb. 5a) und setzt sich aus dem Ergebnisvektor (Patientendatenblock, Wort 229-230) und den Bedingungen (Wort 231-232) zusammen. Die bei der Befundinterpretation verwendeten Einzelinformationen werden wie folgt codiert:

- Die quantitativen Relativprozent-Ergebnisse der Elektrophoresefraktion und das Gesamteiweiß werden anhand ihrer Wertlage graduiert, in semiquantitative Werte umgewandelt und in den Komponenten des Vektors festgehalten.

- Die Form des Pherogramms wird über zusätzliche Auswertemoduln untersucht. Die Ergebnisse dieser Uberprüfung und die oben genannten Patientendaten werden in ja/nein-Form in den "Bedingungen" codiert.

\section{Referenzmuster}

Alle Referenzmuster sind in der sog. Befunddatei abgespeichert (Abb. 5b). Das Referenzmuster setzt sich aus Referenzvektor (Wort 0-2), Bedingungen (Wort 4-5), Bedingungsmaske (Wort 6-7) und 8 Textnummern (Wort 8-15) zusammen. Im Gegensatz zum Ergebnisvektor benötigt der Referenżvektor 3 Datenwörter (Wort 0-2). Die Bedingungen des Referenzmusters (Wort 5-6) und die des Ergebnismusters sind analog aufgebaut. Zusätzlich ist eine sog. Bedingungsmaske (Wort 6-7) angelegt, die im Erkennungsproze $B$ die Selektion der Bedingungen steuert. 


\section{Entecholdungevariablen:}

$N_{1} / N_{2}$ Anzahl dor Poaks

\section{$d_{1}-d_{3}$ Maxima-Mlnimadiferenzen}

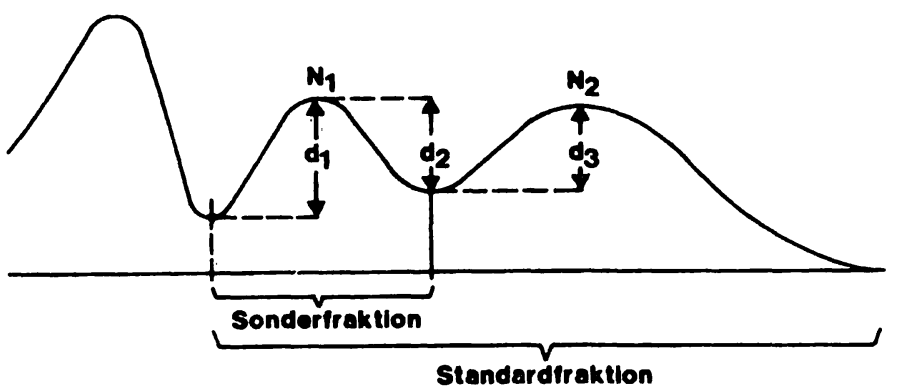

Abb. 3a. Erkennung von M-Peaks in Form von Sonderfraktionen.

\section{Entscheidungsvariablen:}

\section{Albuminabstand \\ W/W. Wendepunkt \\ $\frac{a}{b} / \frac{a+a}{b^{\prime}}$ Höhen-Breitenverhälthis \\ f Fläche}

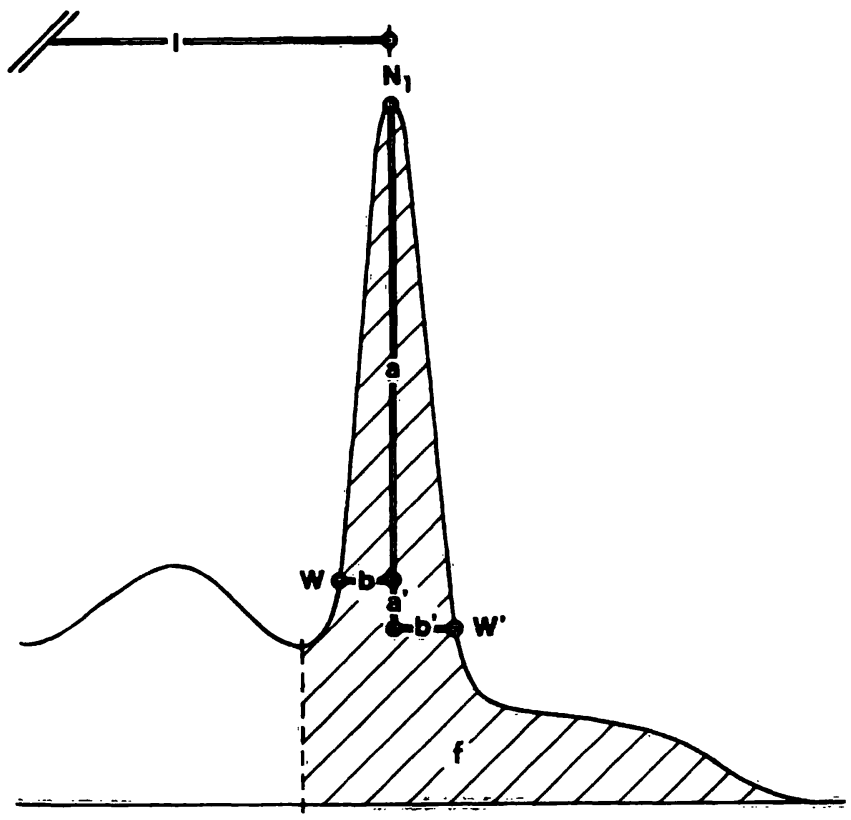

Abb. 3b. Erkennung von überlagernden M-Komponenten.

\section{a Amplitude $\beta$-Fraktion \\ b Höhe des Minimums}

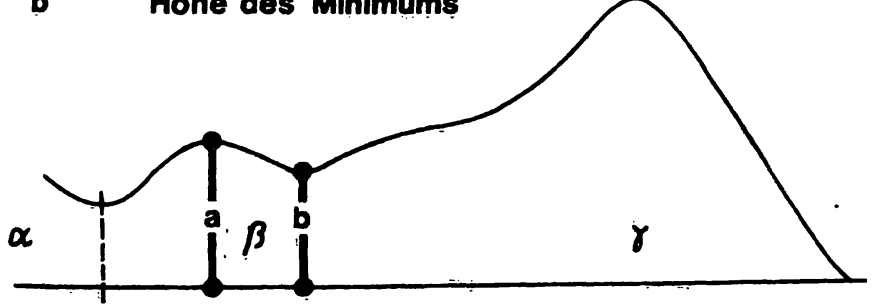

Abb. 4. Entscheidungsvariablen $\beta-\gamma$-Brücke.

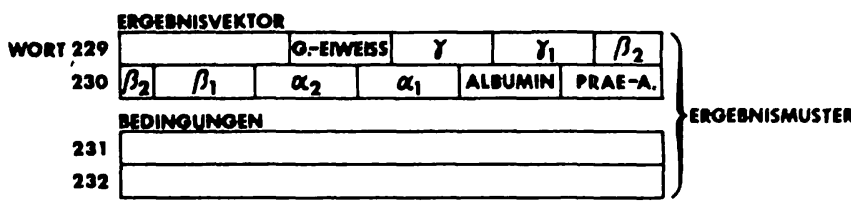

Abb. 5a. Datenstruktur des Ergebnismusters.

(Ausschnitt aus dem Patienten-Ergebnisdatenblock)

Wort 229-230: Referenzvektor

Wort 231-232: Bedingungen

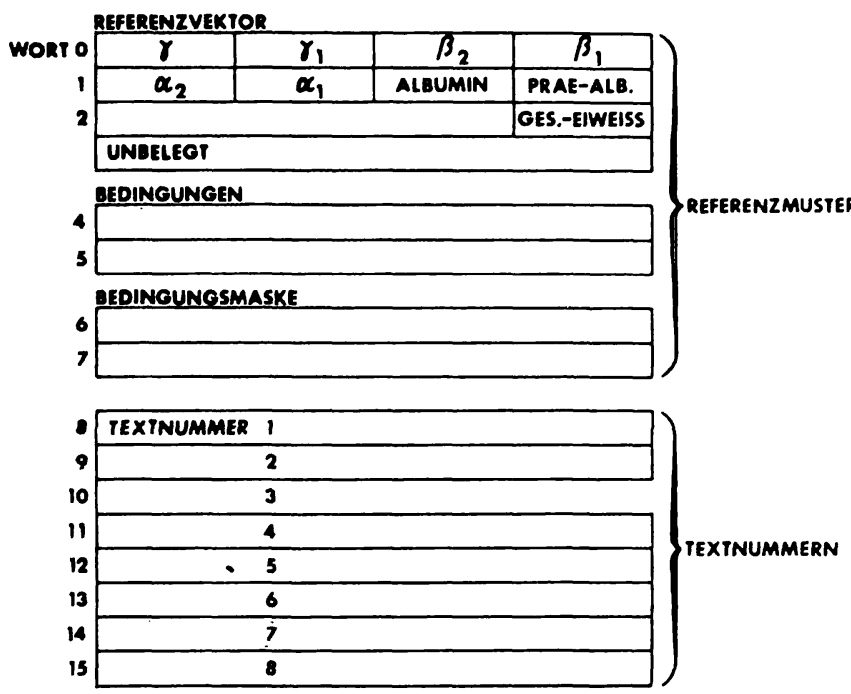

Abb. 5b. Struktur eines Datenblocks in der Befunddatei.

Ein Datenblock (Wort 0-15) besteht aus dem Referenzmuster (Wort 0-7) und 8 Textnummern (Wort 815). Das Referenzmuster ist untergliedert in den Referenzvektor (Wertlage der elektrophoretischen Relativprozent-Ergebnisse und des Gesamteiweißes, Wort 02), die Bedingungen (Wort 4-5) und die Bedingungsmaske (Wort 6-7).

Durch das Ausblenden von Bedingungen, die für ein spezifisches Muster uncharakteristisch sind, wird die Zahl möglicher Referenzmuster verringert. Anhand der Textnummern werden die Textbausteine der Beurteilungen identifiziert.

Codierung des Ergebnis- und des Referenzvektors (rel.\%-Werte der Fraktionen, Gesamteiwei $\beta$ )

Zur Befundmusterauswertung werden als Einzelkomponente die quantitativen Ergebnisse von acht Elektrophoresefraktionen und die Gesamteiweiß-Konzentration in semiquantitative Werte umgewandelt und zu einem neundimensionalen Vektor zusammengefaßt. Jede Komponente des Ergebnisvektors kann den Wert 0 bis 5 (6 Möglichkeiten), jede Komponente des Referenzvektors den Wert 0 bis $F$ ( 16 Möglichkeiten) annehmen. Zur Codierung wird der Meßbereich von 0 bis $\infty$ in 5 Intervalle oder Wertlagebereiche unterteilt. Die Wertzuweisung ergibt sich aus der Zuordnung des quantitativen Fraktions- bzw. des Gesamteiweißwertes zu einem der 5 Intervalle (Abb. 6a, 6b). Die Grenzen der 5 Wertlagebereiche sind entsprechend klinischen Erfahrungswerten festgelegt (Tab. 1), denen die 3s-Grenzen eines Kollektivs klinisch nicht kranker Personen $(n=180)$ zu Grunde liegen. Die Fraktionen $\alpha_{1}$ und $\gamma$ sind im unteren Meßbereich zusätzlich durch eine $2 s$-Grenze unterteilt, um grenzwertige Ergebnisse miteinbeziehen zu können. Um für Albumin und die $\alpha_{2}$ - und $\beta$-Globulin-Fraktion eine bessere Graduierung der Ergebnisse nach klinischen $\mathrm{Ge}$ sichtspunkten vornehmen zu können, wurde für diese Fraktionen nicht das mittlere der 5 Intervalle (Bereich 3) als Referenzintervall gewählt, sondern für Albumin Intervall 4 und für die $\alpha_{2}$ - und $\beta$-Globulin-Fraktion Intervall 2 (Tab. 1). 

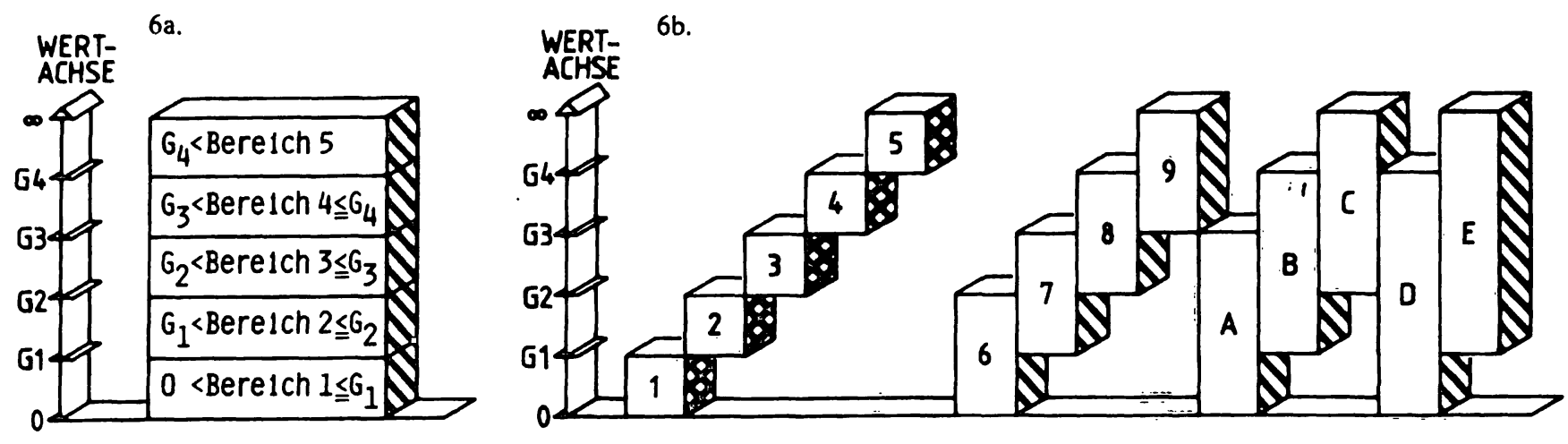

Abb. 6a. Definition der Wertlagebereiche zur Befundmustercodierung.

$\mathrm{G}_{1}$ bis $\mathrm{G}_{4}$ sind die für jede Komponente in die .Parameterdatei eingegebenen Grenzwerte.

Abb. 6b. Wertzuweisung der Vektorkomponenten.

Den Komponenten des Ergebnisvektors werden nur die Werte der zweifach schraffierten Einzelintervalle zugewiesen (1-5), denen des Referenzvektors auch die benachbarten (1-E).

Tab. 1. Bereichsgrenzen der Elektrophoresefraktionen und des Gesamteiweißes für die Befundmustercodierung. Die Einrahmungen stellen die 3s-Grenzen des Referenzkollektivs dar $(n=180)$.

\begin{tabular}{|c|c|c|c|c|c|c|}
\hline \multirow{2}{*}{$\begin{array}{l}\text { Grenz- } \\
\text { wert }\end{array}$} & \multicolumn{6}{|c|}{ Testkomponenten } \\
\hline & $\begin{array}{l}\text { Ge- } \\
\text { samt- } \\
\text { Eiweiß } \\
(\mathrm{g} / \mathrm{dl})\end{array}$ & $\begin{array}{l}\text { Al- } \\
\text { bumin } \\
\text { (rel.\%) }\end{array}$ & $\begin{array}{l}\alpha_{1-} \\
\text { Globu- } \\
\text { line } \\
\text { (rel. \%) }\end{array}$ & $\begin{array}{l}\alpha_{2-} \\
\text { Globu- } \\
\text { line } \\
\text { (rel. \%) }\end{array}$ & $\begin{array}{l}\beta- \\
\text { Globu- } \\
\text { line } \\
\text { (rel. \%) }\end{array}$ & $\begin{array}{l}\gamma- \\
\text { Globu- } \\
\text { line } \\
\text { (rel. \%) }\end{array}$ \\
\hline $\mathrm{G}_{4}$ & 12,0 & 75,5 & 8,0 & 20,0 & 30,0 & 25,0 \\
\hline $\mathrm{G}_{3}$ & 9,1 & 59,3 & 4,4 & 14,0 & 20,0 & 19,5 \\
\hline $\mathrm{G}_{2}$ & 6,0 & 45,0 & 1,6 & 9,9 & 13,1 & 9,8 \\
\hline $\mathrm{G}_{1}$ & 4,5 & 35,0 & 1,0 & 3,7 & 5,7 & 7,8 \\
\hline
\end{tabular}

\section{Wertzuweisung des Ergebnisvektors}

Den Komponenten des Ergebnisvektors wird als Wert direkt die Nummer des Intervalles zugewiesen, innerhalb dessen Grenzen sich die Wertlage des Ergebnisses befindet (Abb. 6a, 6b). Beträgt z.B. die errechnete Albuminfraktion 67 rel.\%, so liegt es im Intervall 4, da das Ergebnis $>G_{3}$ und $\leqslant G_{4}$ ist. Der Komponente "Albumin" des Ergebnisvektors wird daher der Wert 4 zugewiesen. Die Komponente ist " 0 ", wenn die betreffende Fraktion nicht vorhanden ist (Abb. 7). So sind z. B. in unserem System in der "Normal"-Elektrophorese keine $\beta_{2}$ - und $\gamma_{1}$-Globulin-Fraktionen zu finden. Diese treten in der Regel nur bei monoklonalen Gammopathien auf.

\section{Wertzuweisung des Referenzvektors}

Die Komponenten des Referenzvektors geben die möglichen Intervalle an, in denen sich der Meßwert des zu prüfenden Ergebnisses befinden kann, damit die für eine Beurteilung gegebene Bedingung erfüllt ist. Dies können einerseits Einzelintervalle sein, andererseits können bei unserem Vorgehen infolge der hexadezimalen Codierung auch benachbarte Intervalle zusammengefaßt werden (Abb. 6a, 6b, 7).
Zum Beispiel kann'dem Wert „C“ in einer Komponente des Referenzvektors ein Wert des Ergebnisvektors im Wertlagenbereich 5,4 oder 3 entsprechen, damit er dem Referenzmuster als zugehörig erkannt wird. Der Wert " 0 " beinhaltet, da $\beta$ die betreffende Fraktion nicht vorhanden sein darf und „, $\mathrm{F}^{\prime \prime}$, daß die Fraktion bei der Auswertung unberücksichtigt bleibt.

\section{Codierung der Bedingungen}

Im Gegensatz zu den quantitativen Resultaten werden die Beurteilungskriterien, die als Bool'scher Wert ausgedrückt werden können (wahr/nicht wahr), in den „Bedingungen“ festgehalten (Tab. 2). Jeder Einzelbedingung wird:eine von 32 Bitpositionen in einem Doppeldatenwort zugeordnet. Auf diese Weise kann ein direkter Vergleich der Bedingungen des Ergebnis- und Referenzmusters vorgenommen werden. Insgesamt sind 32 Bedingungen möglich, von denen z.Z. 12 (Angaben zur Diagnose, Kurvenform, Vorwerte) ausgewertet werden.

\section{Plausibilitäts-/Vorwertkontrolle}

Da die errechneten Fraktionsintegrale im Labordatensystem archiviert werden, sind Verlaufsbeurteilungen bei erneuter Elektrophoreseanforderung möglich. Aus den Relativvorwerten wird ein Vorwertvektor aufgestellt, der mit dem aktuellen Ergebnisvektor verglichen wird. Weichen Vorwertvektor und Ergebnisvektor in einer Komponente, d.h. einer Elektrophoresefraktion um mehr als 2 Intervallstufen voneinander ab, so wird für den Laborarzt der Hinweis „Vorwertkontrolle“ ausgegeben. Sind die Abweichungen der Einzelfraktionen gegenüber dem Vorwert kleiner als $15 \%$ und zudem beide Vektoren identisch, wird der Befundtext ausgegeben: ,keine wesentliche Änderung zum Vorbefund", wobei wahlweise die der Proteinkonstellation entsprechenden Interpretationshilfen unterdrụ̈ckt wẹrden können.

\section{Angaben zur Diagnose}

In der zweiten Hälfte des Doppelwortes wird entsprechend dèn Angaben auf dem Untersuchungsantrag, die gesicherte Diagnose einer Paraproteinämie oder eines Antikörpermangelsyndroms codiert. Durch diese Einbeziehung der Angaben zur Diagnose werden redundante Beurteilungen vermieden. 


\section{MUSTERCODIERUNG EINER FRAKTION}

\begin{abstract}
ERGEBNISKOMPONENTE (WXHREND ELEKTROPHORESEAUSWERTUNO GENERIERT)
\end{abstract}

WERTLAGE

\begin{tabular}{|r|r|r|r|r|r|r|}
\hline BEREICH 5 & $x$ & & & & & \\
\hline 4 & & $x$ & & & & \\
\hline 3 & & & $x$ & & & \\
\hline 2 & & & & $x$ & & $z$ \\
\hline 1 & & & & & $x$ & \\
\hline OKTALWERT & 5 & 4 & 3 & 2 & 1 & 0 \\
\hline
\end{tabular}

8

REFERENZKOMPONENTE

(ABOESPEICHERT IN BEFUNDDATEI)

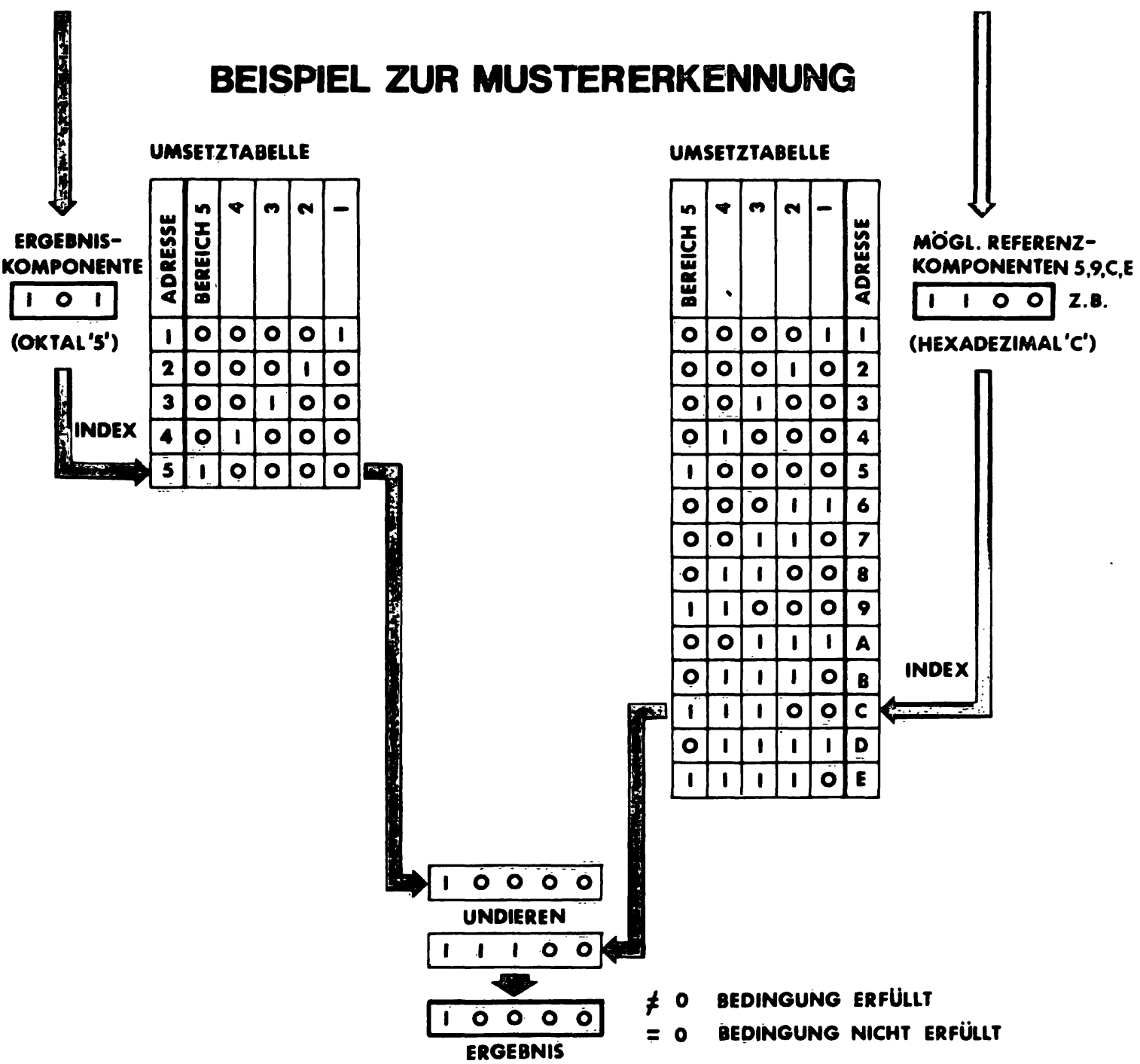

Abb. 7., Beispiel Befundmustercodierung/-erkenung.

\section{Vergleich mit abgespeicherten Referenzmustern}

Ein direkter Vergleich von Ergebnis- und Referenzvektor ist wie bei bisher beschriebenen Verfahren wegen der unterschiedlichen Dimensionen nicht möglich, sondern kann erst nach einer Mustertransformation durchgeführt werden (Abb. 7). Dazu wird sowohl der Ergebnisvektor wie auch der in der Befundmusterdatei gespeicherte Referenzvektor über eine Tabelle umcodiert. Entsprechend den 5 Intervallen sind nach der Umsetzung der jeweiligen Komponente des Ergebnis- bzw. Referenzvektors die 5 Bits zweier Dualzahlen gesetzt oder nicht gesetzt. Beide Zahlen werden in

WERTLAGE

\section{$X$ BEREICH 5}

\begin{tabular}{|r|r|r|r|r|r|r|r|}
\hline$X$ & & & $X$ & & $X$ & & BEREICH 5 \\
\hline$X$ & & $X$ & $X$ & $X$ & $X$ & & 4 \\
\hline & $X$ & $X$ & $X$ & $X$ & $X$ & & 3 \\
& $X$ & $X$ & & $X$ & $X$ & \\
\hline & $X$ & & & $X$ & & \\
\hline 9 & $A$ & $B$ & $C$ & $D$ & $E$ & $F$ & HEX-WERT \\
\hline
\end{tabular}


des Ergebnisses gesetzt werden kann. Die Ergebnisdualzahl nach der „UND“-Verknüpfung ist immer ungleich 0 , sobald nur ein Bit der Ergebnisdualzahl ungleich 0 ist. Eine Beurteilung erfolgt, wenn alle Merkmale von Ergebnis- und Referenzmuster übereinstimmen.

\section{Ausdruck der Befundtexte}

Die zu einem Referenzmuster gegebene Befundung kann aus bis zu acht Textbausteinen zusammengesetzt sein. In der Befunddatei sind nur die Identifikationsnummern der auszugebenden Textbausteine eingegeben. Die Länge eines Bausteines ist auf 128 Zeichen begrenzt; damit ist der erforderliche Platzbedarf auf dem Befundausdruck besser zu erfassen. Die Bausteine können mit einem Sonderzeichen (\$) gekettet werden. Die Einblendung erfolgt formatiert in ein dynamisch der Zeilenzahl angepaßtes Befundfenster.

Tab. 2. Bedeutung der Bedingungen.

Die Anforderungsdaten sind in Bit 0-15, die in zusätzlichen Auswertemoduln ermittelten Bedingungen in Bit 16-31 codiert (vgl. Abb. 5).

Bedingung

(1)

Stationär (ambulant)

Klin. Diagnose Paraproteinämie

Klin. Diagnose Antikörpermangelsyndrom

Doppelalbuminämie vom langsamen Typ

Anodische Albuminverbreiterung

Doppelalbuminämie vom schnellen Typ

Laufzeitdifferenz $\beta-/ \gamma$-Globulin-Fraktion Monoklonaler Peak

$\beta-/ \gamma$-Brücke

Abweichung vom Vorwertvektor $>2$ Stufen

Vorwert $=$ Ergebnis

Vorwert vorhanden
Bit.-Nr.

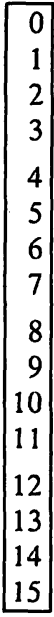

Wort 232

Wort 231

\section{Diskussion der Methodik}

\section{Befundmustercodierung}

Verschiedene Autoren haben Systeme zur rechnerunterstützten Interpretation und Befundgenerierung für die Serumelektrophorese entwickelt. Neben fest programmierten Entscheidungsfolgen (29) sind Systeme beschrieben, die Algorithmen zur Mustererkennung verwenden (2-5). Bereits im Jahre 1968 hat Pribor et al. (3) ein on-line angewandtes Verfahren zur Befundmusterinterpretation vorgestellt. Seine und die später von anderen Autorenn $(2,4,5)$ angewandte Methode basiert auf der Generierung einer Nummer, die aufgrund der Zuordnung des Fraktionsergebnisses zu einem unteren, mittleren oder oberen Wertlagebereich codiert wird und mit einer Zahlenmatrix oder „Wörterbuch" korrespondierender Muster verglichen wird. Die Entscheidungsgrenzen sind jeweils unter Bezug auf Referenzkollektive gesunder Erwachsener festgelegt. Die routinemäßige Anwendbarkeit dieser 3-stufigen Codierung auf andere klinisch-chemischè Kenngrößen, wie Isoenzyme der Lactatdehydrogenase, Schilddrüsenkenngrößen, Elektrolyte und Enzymwerte $(2-4,30)$ wurde beschrieben. Der Nachteil dieses Verfahrens, das einen direkten Vergleich von Befund- und Referenzmuster vornimmt, besteht darin, daß häufig mehrere Muster für eine Beurteilung abgespeichert werden müssen. Die Zahl der möglichen Kombinationen erhöht sich insbesondere dann um ein Vielfaches, wenn von den bislang verwendeten 3 Wertlagebereichen auf 5 Wertlagebereiche erweitert wird, um die Differenzierungsmöglichkeiten $\bar{z} u$ verbessern. Aufgrund des erforderlichen und nicht ökonomisch genutzten Speicherplatzes ist dann die Verwendung einer Interpretationsmustermatrix, wie von Dito (2) beschrieben, nicht geeignet. Zwar können mit dem von Dito beschriebenen Algorithmus auch grenzwertige Ergebnisse interpretiert werden, doch müssen die erforderlichen Permutationen erst durch ein zusätzliches Programm erzeugt werden.

Um diese Nachteile zu umgehen, veriwenden wir einen Algorithmus, der es durch die Transformation des Ergebnis- und Referenzmusters ermöglicht, im Referenzmuster mehrere benachbarte Wertlagebereiche (Intervalle) in nur einem Muster zusammenzufassen. Dadurch und durch die Verwendung einer Bedingungsmaske wird der benötigte Speicherplatz vermindert und die Übersicht über das angelegte Spektrum von Befunden erhöht, da keine Befunddoubletten erforderlich sind.

Ein weiterer Vorzug des zur Befundmustercodierung verwendeten Algorithmus; ist nach unserer Meinung seine einfache Übertragbarkeit. Durch 
Hinzunahme weiterer Vektorkomponenten kann er an die höherauflösenden Elektrophoresetechniken angepaßt werden, die gegenwärtig auf ihre Eignung für klinische Routinebestimmungen untersucht werden. Wegen des hohen Informationsgehaltes der Pherogramme stößt die Interpretation nach visueller Betrachtung an Grenzen. Eine UUbertragungsmöglichkeit auf andere Gebiete der klinisch-chemischen Diagnostik ist gleichfalls gegeben, da die Befundzuordnung nicht mit Hilfe strenger Entscheidungsfolgen, sondern eines Mustervergleichs vorgenommen wird. Gegenüber den bislang beschriebenen Problemlösungen ist die höhere Auflösung des Wertlagebereiches bei gleichzeitiger Reduktion der zu generierenden möglichen Befundmuster von praktischem Nutzen.

Eine bedeutende Verbesserung in der täglichen Routine ergibt sich durch die Integration des HITESystems in das Labordatenverarbeitungssystem des Instituts. Während Pribor et al. (3) und Talamo et al. (5) alle zusätzlichen Informationen manuell off-line erfassen, ermöglicht dieser on-line Anschluß des HITE-Systems in einer Analysenserie eine vollmechanisierte Abarbeitung von 100 Proben mit rechnergesteuertem Befundausdruck einschließlich der Angabe aller demographischen Daten, der Pherogrammkurve und einer Befundung. Daneben kann durch den bidirektionalen Datentransfer Subsystem-Laborrechner das Vorwertmuster in die Befundung miteinbezogen werden. Damit wird einerseits laborintern eine Plausibilitätskontrolle in longitudinaler Form durchführbar und andererseits wird vermieden, daß bei gleichen oder ähnlichen Ergebnissen redundante Interpretationshinweise ohne zusätzlichen Informationsinhalt ausgegeben werden. Die Entscheidung, ob ein Text bei Vorwertübereinstimmung ausgedruckt werden soll - wie z.B. bei anodischen Albuminverbreiterungen -, kann jedoch textspezifisch gefällt werden.

\section{Interpretation der Kurvenform}

\section{Albumin}

Auf die Bedeutung der transitorischen Albuminverbreiterungen wurde vielfach hingewiesen (9-23). Arvan et al. (10) konnten zeigen, daß die Breite des Albuminpeaks proportional zur Höhe der Konżentration von Penicillin und Cephalotin im Serum zunimmt. Damit kann ein Bezug von Toxizität und $\mathrm{Al}-$ buminbreite hergestellt werden. Angaben, ab welchem Grenzwert eine Verbreiterung als pathologisch zu beurteilen ist, liegen nicht vor. Unter Routinebedingungen konnte aber bislang die Albuminbreite quantitativ nicht erfaßt werden, da keine geeigneten, ausreichend präzisen und schnellen Meßgeräte verfügbar waren.

Durch den Einsatz des HITE-System mit Anschluß an ein Labordatensystem wird es möglich, die Albuminbreite in jedem Pherogramm zu vermessen und auf den Befunden einen Hinweis auf mögliche toxische Effekte auszugeben. Gegenwärtig wird dieser Hinweis ausgedruckt, wenn bei einer anodischen $\mathrm{Al}$ buminverbreiterung die Referenzbreite um mehr als $40 \%$ überschritten wird. Damit kann erstmals der Kliniker auf eine im Rahmen der Antibiotikatherapie möglicherweise wichtige Veränderung, die bislang in der Regel nicht erkannt wurde, aufmerksam gemacht werden.

\section{M-Peaks}

Soweit uns bekannt, hat bisher nur Okamura (31) versucht, über Anschluß an eine EDV-Anlage mit Hilfe der 1. und 2. Ableitung der Serumproteinelektrophoresekurve monoklonale Peaks zu erkennen. Ergebnisse zur Effizienz dieser Vorgehensweise wurden nicht mitgeteilt. Mit den von uns bislang festgelegten Parametern wurden bei 83 unausgewählten, immunelektrophoretisch gesicherten Paraproteinen 63 sicher erkannt. Prinzipiell ergeben sich aber auch bei einer rechnerunterstützten Kurvenauswertung die Probleme, die bei visueller Beurteilung der Kurven auftreten. Während hohe monoklonale Peaks leicht erkannt werden, sind schwach ausgeprägte schmalbasige Peaks nur schwer zuzuordnen. Entsprechende Veränderungen können im anodischen Anteil der $\gamma$-Globulin-Fraktion durch Fibrinogen und therapeutisch eingesetzte Immunglobuline, im kathodischen Bereich bei technischen Mängeln durch die Auftragsstelle bedingt sein. Besonders im $\beta$-Globulin-Bereich bereitet die Erkennung eines nur schwach ausgeprägten M-Peaks Schwierigkeiten. Gegenwärtig werden von uns Elektrophoresen von Patienten mit dem klinischen Verdacht auf eine monoklonale Gammopathie vermessen, um nach einer getrennten Auswertung der in der $\beta$ - bzw. $\gamma$-Globulin-Fraktion auftretenden Peaks die Zahl falschnegativer und falschpositiver Befunde weiter zu verringern.

Ausgabe von Interpretationshilfen auf dem Befund

Úber die Form und den Inhalt der rechnerunterstützten Interpretation von Pherogrammen für die klinisch tätigen Kollegen gibt es unterschiedliche Ansichten. Während einerseits die Angabe aller 
möglichen Diagnosen angestrebt wird, um dem Kliniker Hinweise zur Differentialdiagnose zu geben (2, 30), wird andererseits die Meinung vertreten, die Kliniker würden mit langen Listen unbedeutender Diagnosen überhäuft (5), die, wenn sie ungeachtet ihrer diagnostischen Wahrscheinlichkeit mitgeteilt werden, zu unnötigen diagnostischen Folgeuntersuchungen und damit zu Belastungen für den Patienten und erheblichen Kosten führen.

Pribor et al. schlugen vor, zwei „Wörterbücher" zu führen. In dem einen Wörterbuch sollten die Kommentare stehen, die am häufigsten für die betreffende Konstellation zutreffen, in dem anderen die selteneren. Ein entsprechender Vermerk sollte auf den Ursprung der Texte hinweisen. Wir sind der Meinung, daß eine nach Krankheitsbildern klassifizierende Pherogrammbefundung mit der Angabe von spezifischen Diagnosen nur in Einzelfällen möglich ist, wie z. B. Verdacht auf ein $\alpha_{1}$-Antitrypsinmangelsyndrom, Antikörpermangelsyndrom oder monoklonale Gammopathie, in allen anderen Fällen ist eine Beschreibung des elektrophoretischen Musters vorzuziehen, wobei z.T. die Erkrankungen bzw. Krankheitsgruppen angegeben werden, die am häufigsten mit entsprechenden Veränderungen einhergehen. Bislang sind in den Dateien 27 Referenzmuster und dazugehörige Beurteilungen festgelegt. Sie beruhen auf klinischen Erfahrungen und einzelnen Literaturangaben $(24,25,32-37)$. Daneben ist es nach visueller Kontrolle der Befunde möglich, individuelle, in den Dateien nicht festgelegte Texte einzugeben und auf dem Befund auszudrucken.

\section{Kosten}

Das vorgestellte Elektrophoresesystem zur rechnerunterstützten Befundinterpretation von Serumelektrophoresen ist derzeit auf einem Prozeßrechner installiert, der mit einem Minicomputer der mittleren Datentechnik vergleichbar ist. Bei dieser aufwendig erscheinenden Lösung ist $\mathrm{zu}$ berücksichtigen, daß diese Rechnerkonfiguration am Institut vorhanden war, bereits für andere Aufgaben genutzt wurde und sich so für die Integration des HITE-Systems in die bereits bestehende Labordatenverarbeitungsanlage angeboten hat. Zusätzlich ist hervorzuheben, daß die Peakidentifizierung über ein Reférenzserum (7), die damit mögliche standardisierte Speicherung der Ergebnisse und eine die Vorwerte einschließende $\mathrm{Be}$ fundinterpretation eine größere EDV-Anlage erfordert.

Eine Auswertung und Interpretation der Pherogrammkurve allein wäre auch mit mikroprozessorbestückten Auswertegeräten oder mit einem an das Auswertegerät angeschlossenen Kleinrechnersystem zu erreichen. Trotż dieser gegenüber dem vollmechanisierten Analysengerät vergleichsweise kostengünstigen Lösung ist in kommerziellen Geräten bzw. Gerätekombinationen nach unserèr Kenntnis eine weitergehende Auswertung von Pherogrammen und die Ausgabe von Interpretationshinweisen bislang nicht realisiert.

Zusammenfassend ist festzustellen, daß sich das beschriebene Verfahren der Mustererkennung über Mustervergleich und Bedingungsmaske durch eine einfache Codierung und den geringen Speicherplatzbedarf auszeichnet. Durch geringfügige Modifikationen kann es von der Celluloseacetatfolien-Elektrophorese auf höherauflösende Elektrophoreseverfahren oder andere Gebiete der klinisch-chemischen Diagnostik übertragen werden. Für den klinisch tätigen Kollegen ist durch die rechnerunterstützte Interpretation auf dem Elektrophoresebefund sichergestellt, daß keine der wichtigen Informationen, die aus dem Pherogramm abzuleiten sind, verloren geht.

\section{Danksagung}

Für Ihre stets zuverlässige Mitarbeit bei der Durchführung der Untersuchungen sei den medizinisch-technischen Assistentinnen Frl. J. Heberer, Frl. E. Sponer, Frau K. Fatemi, Frau G. Menzel und Frau M. Albiez bestens gedankt.

\section{Literatur}

1. Grassmann, W., Hannig, K. \& Knedel, M. (1951)

Uber ein Verfahren zur elektrophoretischen Bestimmung der Serumproteine auf Filterpapier.

Dtsch. Med. Wochenschr. 76, 333-336.

2. Dito, W. R. (1977)

An octal algorithm for pattern coding and computer-assisted interpretative reporting.

Am. J. Clin. Pathol. 68, 575.

3. Pribor, H. C., Kirkham, W. R. \& Fellows, G. E. (1968) Programmed processing and interpretation of protein and lactic dehydrogenase isoenzyme electrophoretic patterns for computer or for manual use.

Am. J. Clin. Pathol. 50, 67-74.

.4. Ramirez, G., Dinio, R. C. \& Pribor, H. C. (1972)

Pattern recognition of multiple tesțs values as a diagnostic tool.

Comput. Biol. Med. 2, 39-44. 
5. Talamo, S. T., Losos, F. J. \& Kessler, G. F. (1982) Microcomputer assisted interpretative reporting of protein electrophoresis data.

Am. J. Clin. Pathol. 77, 726-730.

6. Fink, P. C. (1981)

A fully automated serum electrophoresis system.

J. Clin. Chem. Clin. Biochem. 19, 379-386.

7. Knüppel, W., Neumeier, D., Fateh-Moghadam, A. \& Knedel, M. (1982)

Einsatz eines vollmechanisierten Elektrophoresegerätes mit rechnerunterstützter Auswertung und Befunderstellung in einem klinisch-chemischen Institut, Teil 1: Systemvoraussetzungen und Methodik.

GIT Lab. Med. 5, 121-128.

Knüppel, W., Neumeier, D., Fateh-Moghadam, A. \& Knedel, M. (1983)

Einsatz eines vollmechanisierten Elektrophoresegerätes mit rechnerunterstützter Auswertung und Befunderstellung in einem klinisch-chemischen Institut, Teil 2: Befundinterpretation.

GIT Lab. Med. 6, 379-384.

18. Fateh-Mogadam, A., Eisenburg, J. \& Lammerz, R. (1969) Alloalbuminämie (Doppelalbuminämie).

Klin. Wochenschr. 47, 976-984.

9. Andre, P., Garre, M., Youinou, P. \& Saleun, J.-P. (1977) Les bisalbuminémies.

Nouv. Presse Med. 6, 4123-4124.

10. Arvan, D.-A., Blumenberg, B.-S. \& Melartin, L. (1968) Transient bisalbuminemia induced by drugs.

Clin. Chim. Acta 22, 211-218.

11. Bismuth, C., Crabie, P. \& Guyon, A. (1976)

Bisalbuminémie transitoire sous béta-lactamines lors d'intoxications phalloidiennes.

Nouv. Presse Med. 5, 1696.

12. Guibaud, S., Bolot, J. F. \& Arnaud, P. (1973)

Bisalbuminémie transitoire chez douze malades soumis à une forte antibiothérapie.

Pathol. Biol. 21, 707-711.

13. Lapresle, C. \& Wal, J.-M. (1979)

The binding of penicillin to albumin molecules in bisalbuminemia induced by penicillin therapy.

Biochim. Biophys. Acta 586, 106-111.

14. Soffiati, G., Pradella, M., Biasioli, B. \& Rigolin, F. (1980) Bisalbuminemia trañisitoria da Penicillina piú Gentamicina. Ann. Sclavo 22, 116-124.

15. Tarnoky, A. L. (1982)

Bisalbuminaemia and albumin variants.

Ärztl. Lab. 28, 314-318.

16. Treut, A., Catheline, M. \& Cloarec, L. (1977)

Bisalbuminémie héréditaire èt familiale. Bisalbuminémie acquise et transitoire.

Pathol. Biol. 25, 47-55.

17. Andre, P., Barra, J.-A., Garre, M., Mioseec, J.-C. \& Saleun, J.-P. (19.77)

Ascite, cirrhose hépatique, bisalbuminémie: faux-kyste du pancréas.

Chirurgie 103, 798-803.

18. Lamotte-Barrilon, S., Nochy, P., Carbon, C., Maillard, J. N., Truchaud, A. \& Nordmann, Y. (1975)

Bisalbuminémie révélatrice d'une fistule pancréatique intrapéritonéale.

Nouv. Presse Med. 4, 2405.

19. Lienhard, Ph., Hay, J.-M. \& Maillard, J.-N. (1978)

Les ascites pancréatiques.

Chirurgie. - Rev. Prat. 28, 379-390.
20. Rousseaux, J. (1976)

La bisalbuminémie au cours des pancréatites: Conditions d'apparition. Implications diagnostiques.

Nouv. Presse Med. 5, 929-930.

21. Rousseaux, J., Debeaumont, D., Scharfmann, A., Pommelet, P., Dautrevaux, M. \& Biserte, G. (1976)

Bisalbuminémies au cours des pancréatites: modifications structurales de la serum-albumin humaine par les enzymes protéolytiques du pancréas.

Clin. Chim. Acta 71, 35-46.

22. Shashaty, G. \& Atamer, M. (1972)

Acquired bisalbuminemia with amylasemia.

Dig. Dis. 17, 59-67.

23. Stoodley, B. J. \& Rowe, D. J. (1970)

Pancreatitis with hyperamylasaemia and bisalbuminaemia. Br. Med. J. 2, 30-31.

24. Hitzig, W. H. (1963)

Die Plasmaproteine in der Klinischen Medizin.

Springer-Verlag, Berlin-Göttingen-Heidelberg.

25. Wuhrmann, F. \& Märki, H. H. (1963)

Dysproteinämien und Paraproteinämien.

Verlag B. Schwabe und Co., Basel-Stuttgart.

26. Langfelder, C., Neumeier, D., Fateh-Moghadam, A. \& Knedel, M. (1978)

Evaluation and diagnostic interpretation of typical electrophoretic diagrams with the aid of a computer. In: Siemaszko, Computing in Clinical Laboratories, pp. 291-298.

The Pitman'Fress, Bath.

27. Bronstein, I. N. \& Semendjajew, K. A. (1973)

Taschenbuch der Mathematik, p. 206.

Harri Deutsch, Zürich-Frankfurt.

28. Fateh-Moghadam, A. (1982)

Die Bedeutung der Immunglobuline $G, A, M$, und $E$ in der Diagnostik von Lebererkrankungen

Der Bayerische Internist 4, 23-27.

29. Nennstil, H.-J. (1976)

Mechanisierte Befundschreibung zur Serumelektrophorese. Ärztl. Lab. 22, 403-406.

30. Swezey, C. B. \& Jacobson, W. (1980)

Computer-based diagnostic reporting for serum electrolytes. Am. J. Clin. Pathol. 74, 812-189.

31. Okamura, K. (1979)

Derivative electrophoretograms and their applications.

Clin. Chim. Acta 96, 273-279.

32. Barandun, S. (1960)

Defektpathoproteinämien.

Symposion: Biochemie und Klinik der menschlichen Bluteiweiße, pp. 203-214.

Benno Schwabe \& Co., Basel/Stuttgart.

33. Kawai, T. (1973)

Clinical aspects of the plasma proteins.

Springer-Verlag, Berlin-Heidelberg-New York.

34. Keyser, J. W. (1979)

Human plasma proteins.

John Wiley \& Sons, Chichester-New York-BrisbaneToronto.

35. Levinson, S. A. \& MacFate, R. P. (1969)

In: Clinical laboratory diagnosis, pp. 503-515, Lea \& Febinger, Philadelphia.

36. Streuli, R. \& Grob, P. J. (1977)

Die Diagnostische Bedeutung des $\alpha_{1}$-Antitrypsins.

Dtsch. Med. Wochenschr. 102, 398-400.

37. Sundermann, F. W., Jr. (1964)

Recent advances in clinical interpretation of electrophoretic fractionations of the serum proteins. In: Sundermann, Serum Proteins and the Dysproteinemias, pp. 323-345.

Pitman Medical Publishing, London.

Dr. med. W. Knüppel

Institut für Klinische Chemie

Klinikum Großhadern

Postfach 701260

D-8000 München 70 
. 